\title{
Robo Advisors: quantitative methods inside the robots
}

\author{
Mikhail Beketov $^{1} \cdot$ Kevin Lehmann $^{2} \cdot$ Manuel Wittke $^{2}$
}

Revised: 13 September 2018/Published online: 27 September 2018

(C) The Author(s) 2018

\begin{abstract}
Robo Advisors (RAs) are perhaps the most important disruptive trend in wealth and asset management today. There is an immense amount of information about RA systems, but still little is known about the core portfolio optimization and asset allocation methods applied within such systems. Thus, to date, there is no comprehensive analysis of the methods used in RAs, their occurrences in these systems, the respective volumes of assets under management (AuM), and the future methodological prospects of the RAs. We analyzed 219 existing RAs worldwide and showed that Modern Portfolio Theory remains the main framework used in RAs. The current trend is to improve and augment this framework rather than applying and developing entirely new approaches. However, we also revealed that the AuM volumes tend to be higher for the systems applying newer and more sophisticated methods. In general, there is a clear gap between the predominant methods applied in RAs and new methodological developments. In the future, as the RA services mature, we can expect that the RAs system will adopt many of the new approaches since they promise good performance and have certain marketing potential.
\end{abstract}

Keywords Robo advisory - Digital asset management . Quantitative methods · Asset allocation methods

Mikhail Beketov

mbeketov@deloitte.de

1 Deloitte GmbH, Berlin, Germany

2 Deloitte GmbH, Dusseldorf, Germany

\section{Introduction}

Currently, Robo Advisors (RAs) are widely recognized as one of the most important disruptive trends in asset and wealth management industry. The terms "Robo-Advise," "Robo Advisor," and "Robo Adviser" have become wellknown buzzwords, and the growth prospects for such systems are highly promising. Thus, different forecasts predict that RAs will globally manage between $\$ 0.8$ and $\$ 8.1$ trillion by 2020 , which is $1-10 \%$ of the total global assets under management (Statista 2017; BI Intelligence 2017). In 2017, the global assets under management (AuM) by RAs comprised approximately $\$ 226$ billion, and the number of users exceeded 12 million (Statista 2017).

The reasons for the current success of RAs and the remarkably optimistic forecasts are diverse. In line with previous research by Deloitte (2016a) and BI Intelligence (2017), the main reasons can be condensed to (i) a new generation of clients, (ii) the advantages of RAs over traditional financial advisors, and (iii) the large-scale financial processes such as the concentration of global wealth and the adoption of RAs in Asia.

In recent years, the wealth management industry encountered a new generation of clients who are receptive to digital technologies, are well educated, prefer to have active and ongoing control over their investments, and rely on the information from multiple (mainly online) sources rather than individual financial advisors. This demographic change is supplemented by changes in older generations that are also becoming increasingly technologically receptive, thereby expecting and demanding digital investment services to be comparable to those provided by tech leaders (Deloitte 2016a). For example, according to BI Intelligence research, $49 \%$ of high-net-worth individuals worldwide (which includes a very small fraction of young 
generations) would consider letting an RA to manage at least some portion of their wealth (Business Insider 2017a). Similarly, research performed by MyPrivateBanking (2017) clearly showed an openness among high-net-worth investors toward RA services.

Regarding the advantages over traditional financial advisors, RA services can be offered at much lower costs in comparison with traditional human advisors. At the same time, they can deliver approximately the same returns on investments as traditional advisors. Therefore, RAs are widely advertised to retail clients. In addition, RAs offer various options to control, customize, and construct investment portfolios from multiple devices (smartphones, laptops, etc.). They also have transparent workflow and monitoring systems, require a low or even no minimum investment, and utilize advanced quantitative methods of portfolio management and optimization. Accordingly, some companies publish detailed methodological whitepapers and scientifically justify their investment strategies (Wealthfront 2017; Scalable Capital 2016).

In a nutshell, an RA can be defined as an automated investment platform that uses quantitative algorithms to manage investors' portfolios and is accessible to clients online. Therefore, the term RA covers a wide range of digital (semi-)automatic investment platforms and services. The general classification of RAs as previously proposed in Deloitte whitepapers (Deloitte 2016a, b) includes four generations. The first- and second-generation RAs comprise online questionnaires and proposals, thereby providing a combination of advice and online access to traditional "manual" asset management services. In contrast, the third and fourth generations of RAs use quantitative methods and algorithms to construct and rebalance the portfolios, thereby performing truly automated portfolio management (the differences between these generations are only the level of automation and methodological advances; for details, see Deloitte (2016a, b). Hence, the third- and fourth-generation RAs are the systems that cover the entire investment/portfolio management process, starting from the selection of the instrument universe and finishing with periodic portfolio rebalancing and appropriate performance reporting.

In the present paper, we consider only third- and fourthgeneration RAs for two reasons. First, in our opinion, only these types of RAs can be considered "true" RAs, as only they really perform portfolio management and optimization (although the terminology is always a subject of debate). Second, the present analyses are mainly focused on the underlying quantitative methods and algorithms, and such analyses make little sense for first- and second-generation RAs. Therefore, the term RA in the following text refers to third- and fourth-generation systems.
Information about RAs currently available online is tremendous and truly diverse in terms of the topics covered and the quality of the materials. However, despite this abundance, little is known about the core portfolio optimization and asset allocation methods applied within the RAs. Thus, there is no comprehensive review and no analyses have been done for the current methodological landscape of the RAs. As a result, the methods, their occurrences in RAs, and the respective AuM volumes remain largely unknown. Furthermore, no analyses have been done regarding the future methodological development of RAs.

The aim of the present study was to gather and analyze the freely available information about the asset allocation and portfolio optimization methods applied in existing RAs worldwide. We have investigated the occurrences of the methods, their combinations, and the respective AuM volumes. In addition, we have considered the possible future methodological solutions and trends.

\section{Materials and methods}

The present analyses were based on a set of 219 systems that could be considered as RAs. This list was compiled during extensive research performed previously by the authors (unpublished database). We analyzed the web pages of these systems and collected all of the information about the asset allocation and portfolio optimization methods. To ensure the quality, the web pages were analyzed twice by different people. The dataset included RAs from 28 countries, with $30 \%$ of the companies located in USA, $20 \%$ in Germany, $14 \%$ in UK, $9 \%$ in Switzerland, and the remaining $27 \%$ in other countries. The RAs in the dataset were founded between 1997 and 2017, with the average founding year being 2014 (the most frequent years are: $2016-48 \%, 2015-16 \%, 2017-15 \%$, and $2014-$ 14\%). The AuM volumes of the analyzed RAs ranged from 1 to 93,000 million USD, with the average and median values being 3,739 and 85 million USD, respectively.

To analyze the frequency of the methods in RAs (i.e., the number of RA systems applying a certain method), we compiled a table in which each RA contributes all of the methods' names mentioned on its webpage (from one to five terms per RA). The table includes all names and definitions used to describe the RA's methodology, regardless of specificity or clarity. Thus, the terms "Modern Portfolio Theory" or "Risk Parity" refer to methodological frameworks that may include other methods that are more specific. Other names, such as "Black-Litterman model" or "Fama-French Factor model," refer to such specific methods. Finally, the terms "Sample Portfolio" and "Constant Portfolio Weights" are defined generally and 
ambiguously, and therefore, the actual methods applied to derive such portfolios are unclear.

An objective, in-depth analysis of this dataset is challenging due to the fuzzy character of the data. Therefore, we performed two separate analyses. First, we identified the main methodological framework of each RA (or a main approach as given on the companies' web pages, considering only one term per RA) and counted the frequencies of such frameworks. Second, we counted the occurrences of all the methods irrespective of their generality and definition clarity (from one to five terms per RA). This latter analysis was aimed at describing and visualizing the overall methodological situation in RAs. We visualized the occurrences with a word-cloud graph. This graph shows the words (i.e., methods names) with the font size being proportional to their frequencies (i.e., methods' occurrences). To make all of the methods visible, the frequencies were $\log (x+15)$-transformed. The graph was created with the online word-cloud generator Wordart (https://wordart.com/).

To understand the AuMs associated with the applied methods, we collected freely available information on the companies' AuMs and linked it to the methods applied by the respective companies. This approach can indicate the AuM volumes "managed" by different methods, but the results are only approximate, as the true information on the methods' AuM is not available. In this analysis, we considered all of the methods' names to cover the entire methodological landscape. For the US companies, the most recent information on the AuMs was retrieved from the Investment Adviser Public Disclosure Web site (https:// www.adviserinfo.sec.gov/). For other companies, the information was collected from the Techfluence database (http://www.techfluence.eu/) as well as the companies' web pages. In the event that a company provides other investment services in addition to RA, the RA-relevant AuM was retrieved.

To understand the entire methodological frameworks of RAs better, we analyzed a small subset of 28 selected RAs and outlined the complete workflow of these systems from the asset universe selection to the performance monitoring and reporting (Table 1). This subset includes the 15 systems that were defined as the best Robo Advisors for 2017 according to BI Intelligence (Business Insider 2017b; all of the companies are from USA) and 13 systems that were defined as most relevant to German investors according to the analyses performed by the Capital journal (Dohms 2017). All of the companies in this latter subsubset are from Germany except for Investify (Luxemburg). The subset of 28 RAs was selected to have a representative sample for the analyses, and we do not share or report any opinions on the quality, importance, or relevance of these RAs.
Table 1 The subset of 28 Robo Advisors selected for the analysis of the complete workflow of such systems

\begin{tabular}{ll}
\hline The Robo Advisors listed by: & \\
\hline Business Insider (2017b) & Capital journal (Dohms 2017) \\
\hline Betterment & Scalable \\
Acorns & Liqid \\
Hedgeable & Visualvest \\
WiseBanyan & Whitebox \\
Wealthfront & Cominvest \\
TradeKing Advisors Core & Quirion \\
SigFig & Vaamo \\
Schwab Intelligent Portfolios & Ginmon \\
Liftoff & Growney \\
TradeKing Advisors Momentum & Fintego \\
FutureAdvisor & Solidvest \\
Personal Capital & Investify \\
Vanguard VPAS & Easyfolio
\end{tabular}

AssetBuilder

Rebalance IRA

The selection includes the 15 and 13 companies mentioned by Business Insider (2017b) and the Capital journal (Dohms 2017), respectively

\section{Results}

\section{Workflow and building blocks of Robo Advisors}

The typical workflow of an RA includes five main steps or building blocks: (i) asset universe selection, (ii) investor profile identification, (iii) asset allocation/portfolio optimization, (iv) monitoring and rebalancing, and (v) performance review and reporting. These steps, of course, differ in detail among the different systems but share the same general traits.

Our analyses based on the selected 28 RAs (Table 1) show that a typical RA's workflow can be described as follows: preselection of ETFs, identification of the client's risk profile and investment goals with online questionnaires, portfolio optimization based on the improved Modern Portfolio Theory approach (Markowitz 1952, 1959), threshold-based rebalancing, and performance monitoring through the web page or a smartphone app.

The first step includes different approaches aimed at (i) creating a representative set of instruments covering different asset classes and types and (ii) selecting low-cost and risk-efficient instruments. The second step may include various models designed to assign a certain risk category to a client based on his/her financial situation, investment goals, age, previous investment experience, and other information (Table 2). The third step, i.e., asset allocation, 
Table 2 Summary of the main building blocks of Robo Advisors

\begin{tabular}{|c|c|c|c|c|}
\hline Asset universe selection & $\begin{array}{l}\text { Investor profile } \\
\text { identification }\end{array}$ & $\begin{array}{l}\text { Asset allocation/portfolio } \\
\text { optimization }\end{array}$ & $\begin{array}{l}\text { Monitoring and } \\
\text { rebalancing }\end{array}$ & $\begin{array}{l}\text { Performance review and } \\
\text { reporting }\end{array}$ \\
\hline $\begin{array}{l}\text { All systems use ETFs with } \\
\text { minor exceptions } \\
\text { including: Mutual/ } \\
\text { Actively Managed Funds, } \\
\text { Sustainable Funds, ETCs, } \\
\text { and Index Funds. } \\
\text { Different selection } \\
\text { criteria include: expense } \\
\text { ratio, total costs, liquidity, } \\
\text { replication method, and } \\
\text { correlation among the } \\
\text { ETFs }\end{array}$ & $\begin{array}{l}\text { Online questionnaires } \\
\text { focused on identifying } \\
\text { clients' risk tolerance as } \\
\text { well as investment } \\
\text { objectives and horizon. } \\
\text { Typically, the questions } \\
\text { are compiled to } \\
\text { understand the objective } \\
\text { risk tolerance through } \\
\text { information on age, } \\
\text { income, savings, } \\
\text { previous investment } \\
\text { experience, and } \\
\text { investment goals }\end{array}$ & $\begin{array}{l}\text { Most of the systems apply } \\
\text { the Modern Portfolio } \\
\text { Theory approach, } \\
\text { supplemented and } \\
\text { modified by various } \\
\text { methods (e.g., Black- } \\
\text { Litterman, VaR and } \\
\text { CVaR optimization }{ }^{\text {a }} \text {. } \\
\text { Notable exceptions } \\
\text { include Risk Parity, Full- } \\
\text { Scale Optimization and } \\
\text { Constant Proportion } \\
\text { Portfolio Insurance. A } \\
\text { few systems apply } \\
\text { constant portfolio weights }\end{array}$ & $\begin{array}{l}\text { Most systems use event/ } \\
\text { threshold-based } \\
\text { rebalancing based on } \\
\text { the daily rebalancing } \\
\text { check. The triggers are } \\
\text { defined as portfolio } \\
\text { structure (i.e., weights), } \\
\text { returns (drift), and VaR } \\
\text { Some systems use } \\
\text { calendar-driven } \\
\text { rebalancing. Some also } \\
\text { use optimized dividend } \\
\text { and cash-flow } \\
\text { (re)investment for the } \\
\text { rebalancing }\end{array}$ & $\begin{array}{l}\text { Half of the systems } \\
\text { provide control and } \\
\text { monitoring possibilities } \\
\text { through the website } \\
\text { only. The half also } \\
\text { provide a smartphone } \\
\text { app. Some systems send } \\
\text { the monthly statements } \\
\text { and quarterly reports } \\
\text { automatically by e-mail }\end{array}$ \\
\hline
\end{tabular}

${ }^{a}$ Many authors and practitioners do not consider VaR and CVaR optimization as an extension of Modern Portfolio Theory, but rather as an alternative to that methodological framework

is the primary focus of the present paper and is analyzed in detail in the next section. The monitoring and rebalancing step may either be event/threshold based with a rebalancing check typically occurring daily or calendar based with predefined rebalancing dates. This step may also include optimized reinvesting of the dividends (and other cash flows) into the assets that are required for the next rebalancing (Table 2). Finally, the performance review and reporting step, as described in Table 2, provides the monitoring options available through web pages and smartphone apps, which may include periodic reports, connections to external accounts, and future profit and risk projections.

\section{Asset allocation methods}

Our analyses of the 219 RAs showed that information about the asset allocation methods is only available for 73 systems. The other systems either do not provide such information or do not use any asset allocation methods (these are the first- and second-generation RAs that do not perform any portfolio optimization; for details, see the "Introduction" section). Therefore, we considered only these 73 RAs for all of the analyses described here. In these 73 systems, we have found the names of 31 various methods.

Analysis of the main methodological frameworks showed that the most frequently applied/mentioned framework is Modern Portfolio Theory followed by Sample Portfolios and Constant Portfolio Weights (Table 3). Among these three terms, only the first can truly be called a quantitative methodological framework, whereas the other
Table 3 Occurrence of different methodological frameworks within the Robo Advisors analyzed

\begin{tabular}{ll}
\hline Methodological framework & Occurrence $(\%)$ \\
\hline Modern Portfolio Theory & 39.7 \\
Sample Portfolios & 27.4 \\
Constant Portfolio Weights & 13.7 \\
Factor Investing & 2.7 \\
Liability-Driven Investing & 2.7 \\
Risk Parity & 1.4 \\
Full-Scale Optimization & 1.4 \\
Constant Proportion Portfolio Insurance & 1.4 \\
Mean Reversion Trading & 1.4 \\
Other & 8.2 \\
\hline
\end{tabular}

two are general definitions provided on the companies' web pages, which may include various methods unknown to us.

Analysis of the entire set of the methods showed similar results. Occurrences of the methods applied in RAs are visualized in a word cloud (Fig. 1). This word cloud includes the names of all of the methods found on the companies' web pages, and the size of the names is proportional to the frequency of their occurrence.

Our analyses of the correspondence between the methods' occurrence and the respective AuMs, as expected, showed that Modern Portfolio Theory has the highest AuM volume (Fig. 2). Remarkably, we also found that relatively advanced methods such as Full-Scale Optimization 
Fig. 1 Word cloud of the occurrence of different methods within the existing Robo systems applying the respective methods). Original frequencies are $\log (\mathrm{x}+15)$-transformed Advisors (i.e., number of

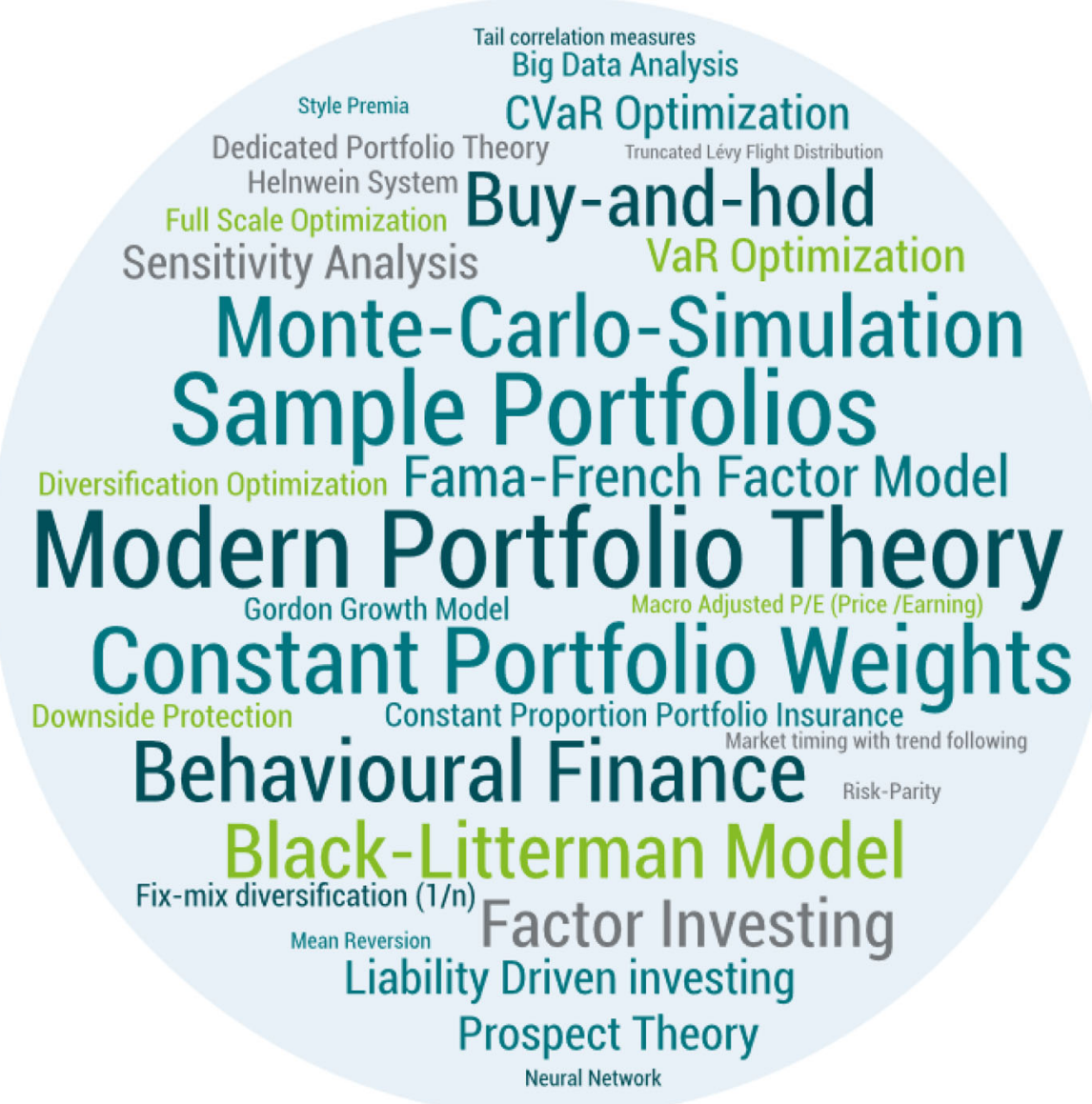

(Cremers et al. 2005; Adler and Kritzman 2007) and the Black-Litterman model (Black and Litterman 1990, 1991) attract high overall AuM volumes despite having relatively low occurrences. In contrast, the simple and generally defined methods such as Sample Portfolios and Constant Portfolio Weights are used more often but attract relatively low AuMs (Fig. 2).

In this analysis, relations between the methods and their hierarchy were not considered, as the entire set of the methods' names was used. Therefore, it has to be mentioned that the Black-Litterman model is used as an additional component within the Modern Portfolio Theory framework. In contrast, Full-Scale Optimization is a methodological framework that is independent of Modern Portfolio Theory.

\section{Discussion}

\section{General remarks}

Our analyses revealed two interesting trends. First, most of the current RAs use Modern Portfolio Theory, and importantly, they tend to improve and augment this framework rather than applying and developing entirely new approaches. Second, the companies using relatively sophisticated methods attract higher AuM volumes despite the fact that these methods are applied less often than the simpler and more generally defined methods. More generally, the overall comparison of the RA methodological landscape with the current state-of-the-art methods shows a clear gap between the methods applied in RAs and newer methods that are widely considered as promising and sci-

\section{Multidimensional improvement of Modern Portfolio Theory}

It is well known that the Modern Portfolio Theory framework has serious practical problems such as extreme input sensitivity, estimation error maximization, and highly concentrated portfolios (Best and Grauer 1991; Michaud 1989; Idzorek 2005). Therefore, in practice, all components of the original Markowitz Mean-Variance Optimization method (Markowitz 1952, 1959) are modified, substituted, and/or complemented by various methods. We define this trend as "Multidimensional improvement of Modern Portfolio Theory." entifically justified in the literature. 


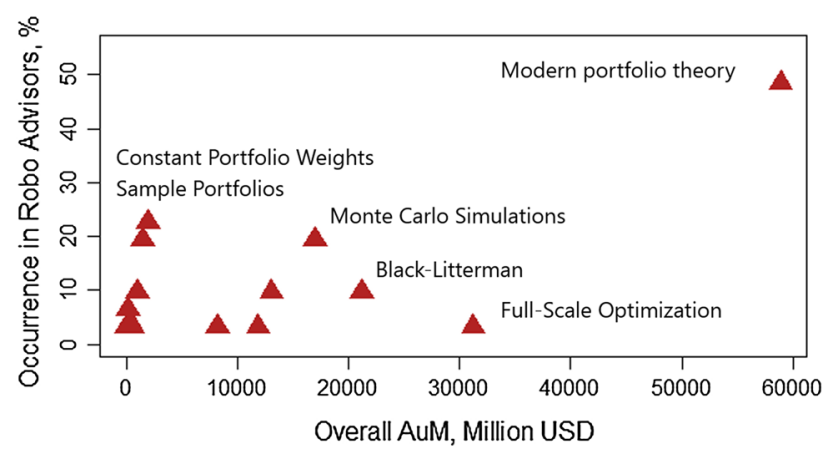

Fig. 2 Relation between the methods' occurrences (\%) and respective volumes of Robo Advisors' assets under management (AuM; million, USD). For clarity, only the names of the methods with either high occurrences or high AuMs are given. Note that each Robo Advisor system contributed between 1 and 5 different methods; there is an overlap of the points in the lower left area of the graph

We have summarized the methods applied to improve the Modern Portfolio Theory framework in RAs in Fig. 3. The methods used to derive the expected returns thus include not only historical averages, which are rarely applied due to high variability and data dependence (Best and Grauer 1991; Michaud 1989; Idzorek 2005), but also the Black-Litterman model (Black and Litterman, 1990, 1991), the Gordon growth model (Gordon, 1959), and CAPM returns (Sharpe 1964). The risk measures include not only the standard deviation, but also value at risk $(\mathrm{VaR})$ and conditional value at risk $(\mathrm{CVaR})$. These approaches are complemented by various portfolio weighting constraints, trading and tax efficiency measures, simulations, different optimization algorithms, and correlation measures (Fig. 3).

These methods, of course, do not comprise a comprehensive list of the methods that are or can be used to improve the Modern Portfolio Theory framework in general, because these are the methods that are currently used for such purposes in RAs. A typical and well-described example of such multidimensional improvement of Modern Portfolio Theory is the framework applied by Wealthfront Investment (2017), which includes many of the methods mentioned above (the methods are described in a detailed whitepaper published online).

The systems combining the methods mentioned in Fig. 3 may deviate considerably from the original Modern Portfolio Theory framework, and it is, of course, a subject of debate whether such combinations represent new approaches or just modifications to Modern Portfolio Theory. The use of the alternative risk functions such as VaR or CVaR, especially, is frequently considered to be an alternative to the Modern Portfolio Theory rather than an improvement of this framework.

\section{Methods of RAs: future prospects}

As mentioned above, our results indicate that, despite being few in number, the companies that use more sophisticated methods attract higher overall AuM volumes (Fig. 2). That also means that there are many small AuM companies on the market offering rather simple and generally defined approaches such as Sample Portfolios and Constant Portfolios Weight.

It is clear that the true methodology behind the various Sample Portfolios systems is unknown to us, and they may actually be rather sophisticated. However, Sample Portfolios RAs have very limited capacity for individualization (i.e., there is no portfolio that is unique to the client). Similarly, RAs using the Constant Portfolios Weight approach may leverage certain complex and well-justified methods used to derive the portfolio weights (which, again, is unknown to us). However, this approach basically offers a single sample portfolio that is rebalanced only to keep the portfolio weights constant.

As described in Introduction, clients investing in RAs are typically receptive to technology and demand both sophisticated as well as individualized digital services and methods. They expect that their investments will be managed with advanced, scientifically justified, modern, and well-implemented methods and technology. This indicates that the sophisticated quantitative methods are and will be used in RAs not only because they promise better performance, but also because they have clear marketing potential. Therefore, some successful RAs publish detailed whitepapers describing and even scientifically justifying their methods (Wealthfront 2017; Scalable Capital 2016; Schwab Wealth Investment Advisory 2017).

It is clear that Modern Portfolio Theory (Markowitz $1952,1959)$ that is more than 65 years old cannot be a very convincing approach for the new generation of investors since they consider cutting-edge methods and technology as a standard in various digitized services. Although any suggestions for the proper methods of portfolio optimization for RAs are beyond the scope of the present paper, we can mention some methods that are suitable for RAs and offer both promising performance as well as a certain marketing appeal. These methods are Risk Parity (Roncalli 2013), Full-Scale Optimization (Cremers et al. 2005; Adler and Kritzman 2007), Scenario Optimization (Adler and Kritzman 2007; Calafiore 2013), and Risk Parity with Skewness Risk (Bruder et al. 2016). The marketing-relevant features of these methods are summarized in Table 4. They indicate that these approaches can be attractive to investors expecting at least some level of sophistication in RAs' methodology and technology.

Given the mismatch between the current RAs' methodology and the state-of-the-art methodological 


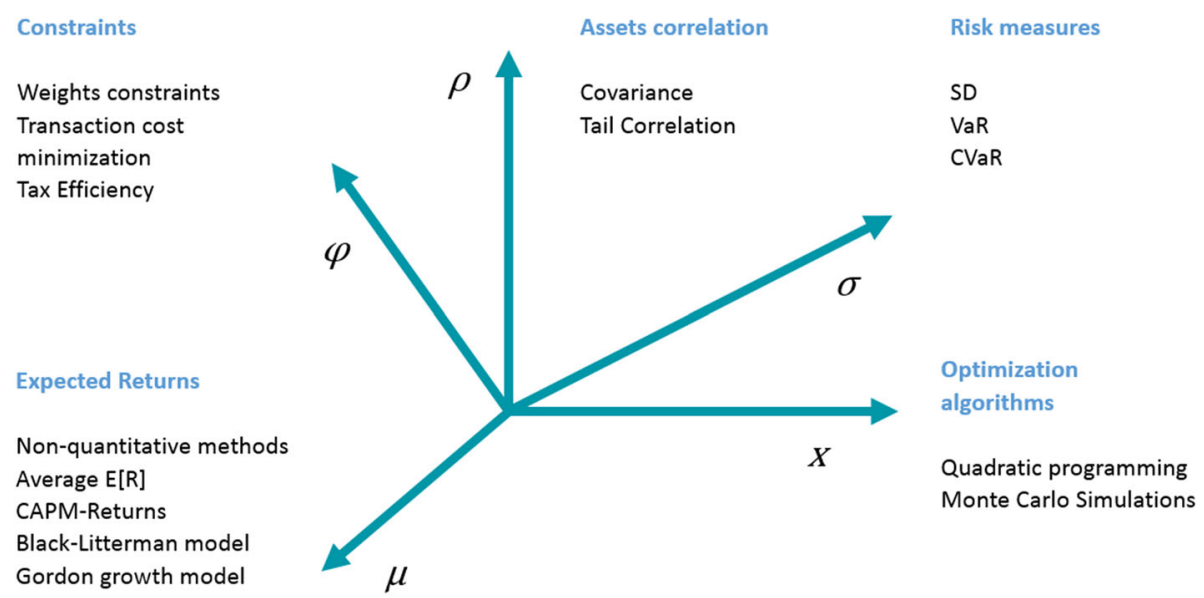

Fig. 3 Schematic of the "Multidimensional improvement of Modern Portfolio Theory" in Robo Advisors. The methods mentioned are those that used in RAs, and they do not comprise a comprehensive list of the methods that are or can be used to improve the Modern
Portfolio Theory framework in general. Note: VaR and CVaR optimization are frequently considered to be alternatives to Modern Portfolio Theory rather than improvements to this framework

Table 4 Marketing-relevant features of several methods that represent a promising alternative to the Modern Portfolio Theory framework and can be used in Robo Advisors

\begin{tabular}{ll}
\hline Method & Marketing-relevant features \\
\hline Risk Parity & $\begin{array}{c}\text { Risk Parity offers perfect or "perfectionistic" risk diversification since all of the portfolio components have } \\
\text { identical risk contributions } \\
\text { Full-Scale Optimization offers customizable, client-specific utility functions and can consider the risk of } \\
\text { catastrophic loss }\end{array}$ \\
$\begin{array}{l}\text { Scenario Optimization } \\
\text { Risk Parity with Skewness } \\
\text { Risk }\end{array}$ & $\begin{array}{l}\text { Risk Parity with Skewness Risk has all of the properties of the Risk Parity but also considers the risk of catastrophic } \\
\text { loss }\end{array}$ \\
\hline
\end{tabular}

developments, together with investors' expectations, we can expect serious changes in the RA sector. The newer methods, which are more sophisticated and capable of individualization, will be widely introduced in RAs, where they will be applied as vehicles to improve performance and as marketing tools used to attract new investors. The methodological competition among market participants will dramatically increase as RAs' services mature, and the sector becomes saturated with diverse offers and companies.

The future development and growth of RAs will depend on many factors. The key question for the growth of the entire RA sector is whether and how wealthy investors will invest in RAs. Existing studies show openness among such investors toward RAs, but they would prefer to have some hybrid models that allow certain human control over the investment process (MyPrivateBanking 2017). More generally, hybrid models are now frequently considered to be the next trend in the RA sector, as investors would like to have human control at least during unusual or crisis situations (Business Insider 2017c). Regarding the asset allocation and portfolio optimization methods, we expect that the methodological core of such hybrid systems will be similar to the methods used in "pure," fully automated RAs. The only expected difference is the introduction of additional components required for both the manual adjustment of the strategies and the inclusion of certain "external" information and analytical opinions/views (similar to the Black-Litterman model already used in existing RAs).

Open Access This article is distributed under the terms of the Creative Commons Attribution 4.0 International License (http://crea tivecommons.org/licenses/by/4.0/), which permits unrestricted use, distribution, and reproduction in any medium, provided you give appropriate credit to the original author(s) and the source, provide a link to the Creative Commons license, and indicate if changes were made. 


\section{References}

Adler, T., and M. Kritzman. 2007. Mean-variance versus full-scale optimisation: In and out of sample. Journal of Asset Management 7(5): 302-311.

Best, M.J., and R.R. Grauer. 1991. On the sensitivity of meanvariance-efficient portfolios to changes in asset means: Some analytical and computational results. The Review of Financial Studies 4: 315-342.

BI Intelligence. 2017. The robo-advising report: Market forecasts, key growth drivers, and how automated asset management will change the advisory industry. https://www.businessinsider.com. au/the-robo-advising-report-market-forecasts-key-growth-dri vers-and-how-automated-asset-management-will-change-theadvisory-industry-2016-6. Accessed 23 Feb 2018.

Black, F., and R. Litterman. 1990. Asset allocation: Combining investors views with market equilibrium. Fixed income research. Goldman, Sachs \& Company.

Black, F., and R. Litterman 1991. Global asset allocation with equities, bonds, and currencies. Fixed income research. Goldman, Sachs \& Company.

Bruder, B., N. Kostyuchyk, and T. Roncalli. 2016. Risk parity portfolios with skewness risk: An application to factor investing and alternative risk premia.

Business Insider. 2017a. Is Robo Investing better than traditional investing? See the pros and cons. http://www.businessinsider.de/ 4-reasons-robo-investing-growing-2017-1?r=US\&IR=T. Accessed 23 Feb 2018.

Business Insider. 2017b. Here are the top 15 Robo Advisors making stock portfolio management easy in 2017. http://www.busines sinsider.de/best-robo-advisors-2017-1. Accessed 23 Feb 2018.

Business Insider. 2017c. Robo Advisors vs. human financial advisors: Why not both? https://www.businessinsider.de/hybrid-robo-advi sors-will-manage-10-of-all-investable-assets-by-2025-2017-1121 ? $=$ US\&IR=T. Accessed 4 May 2018.

Calafiore, G.C. 2013. Direct data-driven portfolio optimization with guaranteed shortfall probability. Automatica 49(2): 370-380.

Cremers, J.H., M. Kritzman, and S. Page. 2005. Optimal hedge fund allocations. Journal of Portfolio Management 31(3): 70-81.

Deloitte. 2016. The expansion of Robo Advisors in wealth management. White Paper.

Deloitte. 2016. Robo Advisory in wealth management. White Paper.

Dohms, R. 2017. Gewinnmaschinen. Capital 8: 116-123.

Gordon, M. 1959. Dividends, earnings, and stock prices. The Review of Economics \& Statistics 41: 99-105.

Idzorek, T. 2005. A step-by-step guide to the Black-Litterman model: Incorporating user-specified confidence levels. Research Paper.

Markowitz, H. 1952. Portfolio selection. Journal of Finance 7: 77-91.

Markowitz, H. 1959. Portfolio selection: Efficient diversification of investment. New York: Wiley.

Michaud, R.O. 1989. The Markowitz optimization enigma: Is optimized optimal? Financial Analysts Journal 45: 31-42.
MyPrivateBanking. 2017. Wealthy clients open to robo-advisors but still want the human touch. http://www.myprivatebanking.com/ article/report-affluent-and-wealthy-investors-attitudes-towardsrobo-advisors-2017. Accessed 4 May 2018.

Roncalli, T. 2013. Introduction to risk parity and budgeting, Chapman \& Hall/CRC financial mathematics series. Boca Raton: CRC Press.

Scalable Capital. 2016. The scalable capital investment process. White Paper.

Schwab Wealth Investment Advisory. 2017. Schwab intelligent portfolios $^{\mathrm{TM}}$ asset allocation white paper. https://intelligent. schwab.com/public/intelligent/insights/whitepapers/asset-alloca tion.html. Accessed 23 Feb 2018

Sharpe, W.F. 1964. Capital asset prices: A theory of market equilibrium. Journal of Finance 19: 425-442.

Statista. 2017. Statistical portal, Robo Advisors. https://www.statista. com/outlook/337/100/robo-advisors/worldwide\#marketStudy. Accessed 23 February 2018.

Wealthfront. 2017. Wealthfront investment methodology white paper. https://research.wealthfront.com/whitepapers/investment-metho dology/. Accessed 23 Feb 2018.

Mikhail Beketov is a senior manager in the quant team within Deloitte's service line Financial Risk Solutions, Risk Advisory. He is also a lecturer of financial econometrics at the Frankfurt School of Finance and Management. His main areas of expertise are quantitative portfolio and risk management, investment and trading strategies, and financial econometrics. Mikhail has more than 14 years of experience as a research scientist and quantitative researcher/analyst in the asset management industry and consulting. He earned his Diploma and Ph.D. at the Novosibirsk State University, Russia, and his Master's degree in Quantitative Finance at the Frankfurt School of Finance and Management, Germany.

Kevin Lehmann is a consultant in Deloitte's quant team within Deloitte's service line Financial Risk Solutions, Risk Advisory. He is involved in various projects as an expert in stress-testing, the valuation of financial instruments, numerical methods and portfolio optimization. Kevin earned his Master's degree in Business Mathematics at the Technical University of Dortmund, Germany.

Manuel Wittke is a senior manager in quant team within Deloitte's service line Financial Risk Solutions, Risk Advisory. He coordinates and implements various projects focusing on portfolio risk simulation (including market and counterparty credit risk), robo advisors, derivatives pricing models and portfolio benchmarking. He established Deloitte's own pricing library and is currently leading the quantitative Robo-Advisory group together with Mikhail Beketov. Before his career at Deloitte, he was a financial engineer in Postbank's Quantitative Analytics team. Manuel earned his Diploma and Ph.D. in Financial Economics at Bonn University, Germany. 